\title{
VLF Noise Bands Observed by the Alouette I Satellite
}

\author{
J. S. Belrose and R. E. Barrington \\ Defence Research Telecommunications Establishment, Radio Physics Laboratory, Ottawa, Ontario, Canada
}

(Received May 28, 1964; revised August 26, 1964)

\begin{abstract}
Alouette I observations of VLF ionospheric noise or hiss have revealed some interesting features of such noises when received within or above the ionosphere. In addition to emission bands whose frequencies are relatively independent of position, or vary sporadically with position, the satellite often sees an emission band (or bands) with a well defined lower frequency that increases with decreasing magnetic latitude of the satellite. On occasion similar emissions are "triggered" or enhanced by short and long fractional hop whistlers propagating past the satellite, which places the generation region in the vicinity of the satellite. The most regular noise bands are found in a rather narrow zone defined by magnetic $L$ values between 2.5 and 4 , invariant magnetic latitudes 50 to $60^{\circ}$, but bands with a stronger dependence on the magnetic field are sometimes found at higher latitudes. Observations during full sweeps of the satellite across the magnetic equator have revealed that the variable frequency band seen in the Northern Hemisphere can sometimes be repeated at almost exactly corresponding $L$ values in the Southern Hemisphere.

At the time of major noise storms, the auroral zone js sometimes well defined by the observations, as lying between $L$ values of 4 to 9 , magnetic latitudes 60 to $70^{\circ}$.

It is not possible at present to give any final explanation of these noise bands, but certain of their features are discussed in the light of current theories of the generation of VLF ionospheric noise.
\end{abstract}

\section{Introduction}

Bursts of VLF ionospheric noise or "hiss," which occur in the audio frequency range 0.1 to $15 \mathrm{kc} / \mathrm{s}$, and last for periods ranging from several minutes to a few hours, have been studied at DRTE and elsewhere [Ellis, 1959] and Watts et al. [1963], for several years. Hiss occurs in broad bands or in one or more narrow bands. The narrow bands often have bandwidths of $1 \mathrm{kc} / \mathrm{s}$ or less. The most common type, "isolated bursts," is typical of magnetically quiet conditions. These are also sometimes associated with isolated magnetic bays, and ionospheric radio wave absorption. A second type occurs as an extended series of hiss bursts or noise storms. All major magnetic storms are accompanied by noise storms. Hiss has been correlated with airglow [Duncan and Ellis, 1959], with aurora [Martin et al., 1960], and with abnormal $D$-region absorption [Watts et al., 1963].

Although the ground observations have revealed much information on the occurrence of the hiss bursts, there are difficulties in interpreting detailed studies of their characteristics. This is partly because ionospheric attenuation affects the noise intensities observed by a ground-based receiver, and partly because propagation under the ionosphere allows observation of hiss generated in a wide range of magnetic latitudes. The effects of the lower ionosphere on the propagation of VLF emissions can be largely eliminated, which thus simplifies the study of these emissions, by using satellite-borne receivers. In addition a receiver in a satellite, above or within the ionosphere, allows investigation of hiss more closely associated with generation and propagation on a particular line of force, and in satellites with a high inclination orbit, a whole range of magnetic latitudes can be studied in a fairly short interval of time.

On September 29, 1962, the Alouette I satellite was launched into orbit, and, although primarily designed as a top-side sounder, a broad band (0.5 to $10 \mathrm{kc} / \mathrm{s}$ ) VLF receiver was included in the pay load. The satellite moves in a nearly circular orbit at an altitude of $1025 \pm 20 \mathrm{~km}$ and has an inclination of $80^{\circ}$ to the equator. This allows observation of the VLF spectrum over a wide range of latitudes, while the nearly circular orbit simplifies the interpretation of the results, e.g., over North America the height of the satellite can for most purposes be considered constant at $1.16 \pm 0.02$ earth radius and the position of the spacecraft in the magnetic field can therefore very simply be determined. It is the purpose of this paper to discuss some of the satellite observations of VLF emissions, and to compare these observations with present theories of the generation of VLF noise. Although this paper gives more detail than the preliminary note which has already been published [Barrington et al., 1963], since the analysis and interpretation of these data are still being done, this paper can only describe the experimental facts insofar as they are presently known. 


\section{Observations}

In this paper the data are sorted according to the magnetic parameter $L$ [McIlwain, 1961]. The parameter $L$ is constant along a line of force and labels the magnetic shell on which an electron bounces in latitude and drifts in longitude. Numerically $L$ is such that for a perfect dipole field, it would be the equatorial radius to a magnetic shell, expressed in units of earth radii. In comparison of the observations with current theories of the generation of VLF noise (sec. 5), it will be necessary to refer to geomagnetic latitude. In the case of a perfect dipole, the magnetic latitude measured to the point where the line of force passing through the satellite cuts the earth's surface $(R=1)$ is

$$
\Lambda=\cos ^{-1} \sqrt{1 / L}
$$

$L$ is however calculated from the real magnetic field as approximated by Finch and Leaton [1957], and therefore $\Lambda$ is calculated simply from $L$ as indicated above. The discrepancy between $\Lambda$ and geomagnetic latitude is at most about $2^{\circ}$ over North America.

Figure 1 shows the track of an Alouette pole-topole pass recorded once per week, which gives full coverage from $80^{\circ} \mathrm{N}$ to $80^{\circ} \mathrm{S}$ latitude. Other passes, not so complete in continuous coverage, are recorded at other times (about $2 \frac{1}{2} \mathrm{hr}$ of recordings are made each week); e.g., a pass extending from NE of Bermuda, over Newfoundland, passing midway between Narsarssuak and Godhavn, and ending $\mathrm{N}$ and $\mathrm{E}$ of Spitsbergen, is recorded in cooperation with ground recordings made by the Danes; and a recording beginning over the Queen Charlotte Islands and ending at about $20^{\circ} \mathrm{N}$ latitude over the Pacific is recorded at Stanford for comparison with ground recordings made there; and two recordings each week are made for satellite passes over the SE coast of Australia for comparison with ground recordings made at Hobart and Brisbane. The purpose of this figure is to show the asymmetry of the satellite track with respect to the magnetic field. The contours are constant magnetic $L$ values at $1025 \mathrm{~km}$ (the satellite height). The pass shown covers a range of $L$ values from about 60 in the north to about 7 in the south.

A surprising feature of the signals observed by the satellite VLF receiver are bands of noise which often show systematic variations with the position of the space craft in the geomagnetic field. Figure $2^{1}$ shows one of the early examples of ionospheric noise which exhibited systematic changes in the spectral distribution of noise with satellite latitude (this event was reported in the paper by Barrington and Belrose, 1963). In this example the noise is

1 The spectrograms shown in this paper are not consistent, in that signal is shown as dark lines or bands in figures 2 and 7 , but white in figures 3,4 , and 5 .

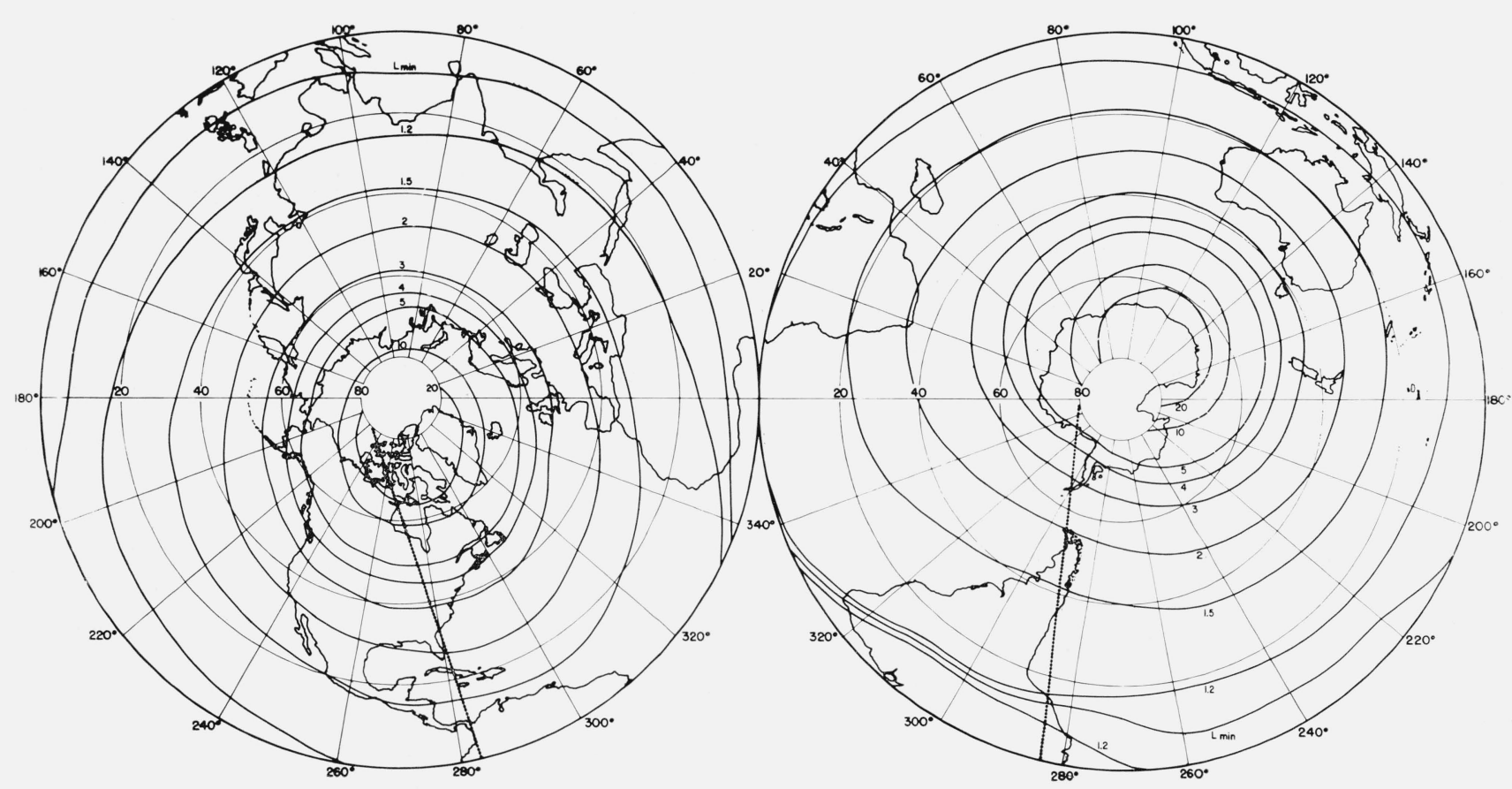

Frgure 1. Polar map showing contours of constant L at $1025 \mathrm{~km}$, and the track of particular satellite pass which recorded VLF data discussed in this paper. 
sporadic but shows a systematic tendency for the lowest frequency at which noise is found to decrease as the satellite moves to high latitudes, over a range of $L$ between about 2.5-3.5. Above this latitude sporadic noise is found throughout the entire frequency range of the VLF receiver with no well defined lower limit. Figure 3 is another example, but shows two bands of sporadic noise observed over the same range of $L$ values, both of which decrease as the latitude of the satellite increases. Figure 4 is an example recorded at higher latitudes.

Figure 5 is an interesting event, recorded on 31 January 1963 , from 1057 to 1148 UT, while the satellite passed through its full range of latitude. A narrow noise band was observed, the low limit of which changed from about $2 \frac{1}{2}$ to $9 \mathrm{kc} / \mathrm{s}$ as the satellite moved from $L$ values of about 3.5 to 2.5. To a very high degree the noise observed at the same $L$ value in the northern and southern hemisphere was the same in spite of the facts that the observations in the two hemispheres are separated by at least 30 min in time and several degrees in longitude, and that the local gyrofrequencies at the points differ by as much as 30 percent. These facts suggest that such noise bands must be due to conditions in the exosphere which are relatively stable and uniform over a large region.

Other features seen in figure 5 worthy of discussion are:

(1) The relatively constant noise bands from the lower edge of the receiver pass band (about $400 \mathrm{c} / \mathrm{s}$ ) to $2.5 \mathrm{kc} / \mathrm{s}$ which were observed when the vehicle was in a region where the magnetic parameter $L$ was greater than 9 .

(2) Similar noise bands were found in the region where $L$ was less than 2.5 (even at the equator, not shown, where $L_{\mathrm{min}} \simeq 1.1$ ).

(3) In the region where $L$ lies between 9 and 4 , the noise was very erratic both in amplitude and frequency.

One of the striking observations of Alouette is the appearance of VLF emissions below $1 \mathrm{kc} / \mathrm{s}$ at virtually all latitudes. On the ground such emissions are usually observed only at high latitudes. Their observation at all latitudes in the satellite indicates that the emission mechanism is effective over a wide range of magnetic latitudes, and the absence of observations on the ground must be attributed to the effects of the lower ionosphere on the propagation of the VLF emissions. The region of irregular ionospheric noise, in the range where $L$ lies between 9 and 4 of figure 5, is certainly associated with the auroral zone.

We have not analyzed enough examples of noise bands to come to any firm conclusions about the morphology of such disturbances, but a few comments, particularly in regards to the disturbance pattern of hiss bands shown in figure 5 can be made. Ground station recordings of VLF noise made at Ottawa $(L \simeq 3.6)$ on a slowly moving paper chart record, employing narrow band receivers centered on 7,4 , and $1 \frac{1}{4} \mathrm{kc} / \mathrm{s}$, and an asymmetrical detector circuit to minimize effects on the recordings due to

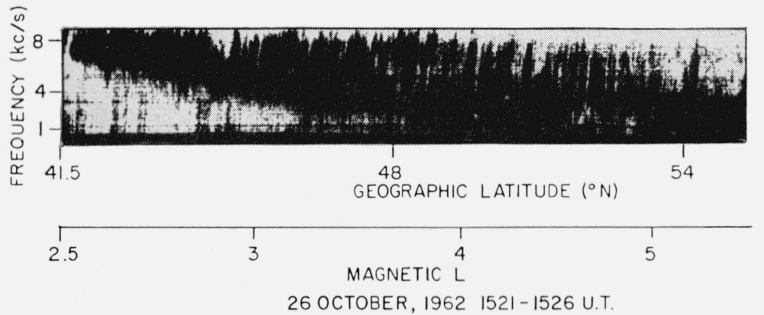

Figure 2. Example of ionospheric noise which exhibits systematic changes in the spectral distribution of the noise with satellite latitude.

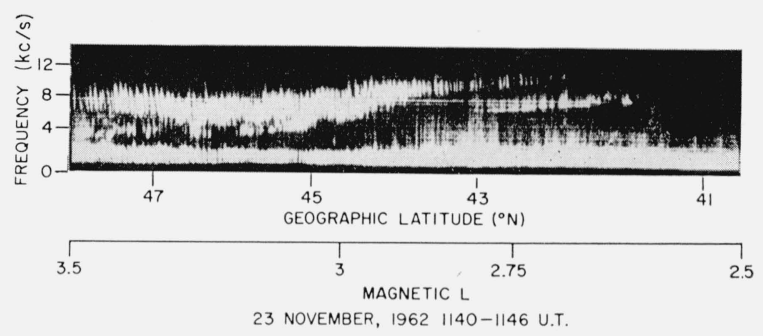

FiguRE 3. Example of ionospheric noise which exhibits systematic changes in the spectral distribution of the noise with satellite latitude.

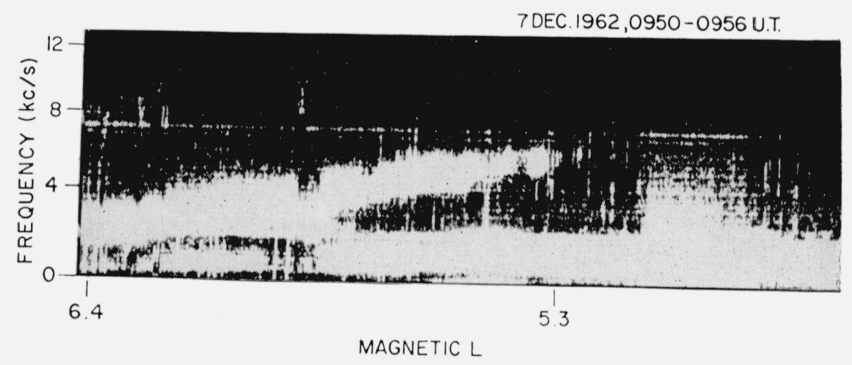

FIgure 4. Example of ionospheric noise which exhibits systematic changes in the spectral distribution of the noise with satellite latitude.
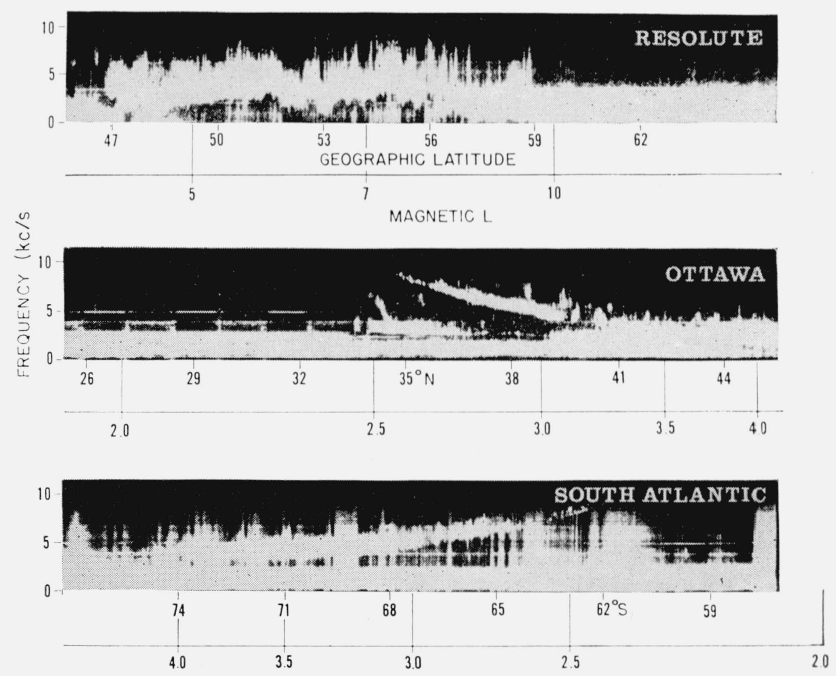

Figure 5. Spectrogram of VLF ionospheric noise observed in Alouette I on 31 January 1963, 1057-1148 UT.

The track of the satellite for this recording is shown in fig. 1. The captions on the three segments shown refer to the telemetry station where the data were recorded (the equatorial segment is not shown). 


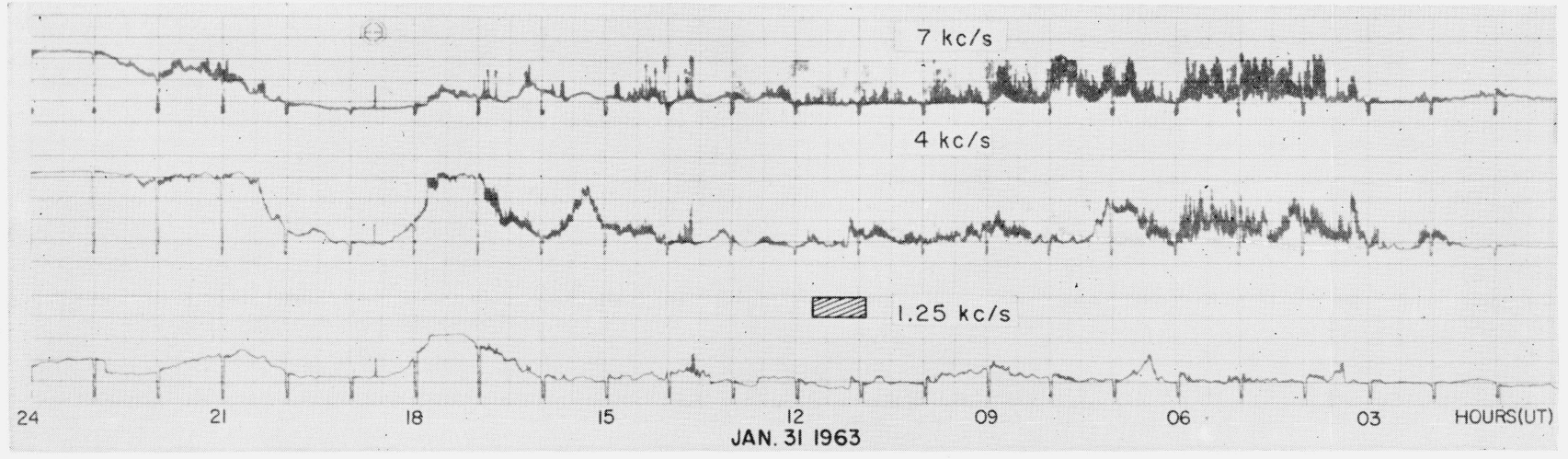

FIGURE 6. Recording of VLF hiss made on three narrow band noise burst receivers at Ottawa $(\mathrm{L}=3.6)$ at the freauencies shown. Time marks are put on the record at hourly intervals. The base line for no (or little) noise can be seen at the beginning of the recordings.

atmospherics are shown in figure 6 . It is clear that the satellite recording, made at the time shown by the cross-hatched rectangle, was made during a series of noise bursts (in fact noise bursts of much greater amplitude were recorded before and after the making of the satellite record). This series of noise bursts began, after a period of magnetic and VLF noise calm, at about 09 U'T on 30 January, and continued in varying degrees of intensity until about 04 U'T on 2 February. This noise storm was associated with a geomagnetic disturbance observed in local and planetary $k$-indices. It seems likely that the electron streams causing the geomagnetic disturbance also produce the hiss, although an experiment to test any possible detailed correlation has not yet been done. A satellite VLF recording made at 0044 to 0049 on 1 February 1963, while Alouette passed through a range of $L$ values from about 2.5 to 6 (the satellite track passed over the Great Lakes area to the west of Ottawa), is also pertinent to our discussion of the 30 January 1963, noise storm. Although magnetic activity was less at this time, ground recording's of VLF hiss at Ottawa $(L \simeq 3.6)$ showed greater activity (see fig. 6 which shows records up to just before the making of the satellite recording). The satellite observations did not show on this occasion the two zones clearly evident in the earlier recording (fig. 5); viz, the noise band whose frequency changes with latitude in the range where $L$ is between 2.5 to 3.5 , and the auroral disturbance zone where $L$ is between 4 to 9 . The satellite observations showed two quasi constant noise bands for $L$ between 3 to 6 . The upper one $(2.5$ to $4 \mathrm{kc} / \mathrm{s})$ was less regular and weaker.

These results, as well as more detailed comparisons between ground station observations and satellite data reveal that there is no close correlation. This may be due to absorption or total internal reflection of these noisy signals in the ionosphere, the noise arising only in the immediate vicinity of the satellite (see sec. 3 for further discussion), or due to the fact that the ground stations can observe VLF signals which have emerged from the ionosphere over a large area.
As mentioned above detailed correlations between particle streams (of the appropriate energy range) and VLF hiss have not vet been made. It is unfortunate that with the Alouette I satellite the VLF receiver and the particle detector recorders are not operated simultaneously. Nevertheless comparisons can be made between the VLF data and average properties of the intensity distributions for particles. The two places where interesting features are sometimes found in VLF data, viz, $L$ between 2.5 to 3.5 and $L$ between 4 to 9 , are also places where features are found in the intensity distribution of trapped (and dumped) electrons. McDiarmid et al., (1963) have shown that the intensity distribution of trapped electrons (in the lowest energy range observed, viz, $E>40 \mathrm{kev})$ observed by Alouette exhibits two maxima separated by a minimum at $L=3.2 \quad(\Lambda=$ $\left.56^{\circ}\right)$. The minimum is not always present. Large fluctuations occur around $L=2.2\left(\Lambda=48^{\circ}\right)$, the approximate position of the lower latitude peak (in the so-called slot between the inner and outer radiation belts). The second peak at $L=5.6\left(\Lambda=65^{\circ}\right)$ corresponds approximately with a peak found in the average intensity of precipitated electrons having similar energies (which occurs at $L=6.5\left(\Lambda=67^{\circ}\right)$ ). Thus the VLF noise bands which exhibit a systematic change with the earth's field are observed at a latitude near the slot between the inner and outer radiation belts, while the higher latitude disturbance zone corresponds approximately with $L$ values at the edge of the magnetosphere, and the horn of the outer Van Allen belt.

\section{Triggering or Enhancement of VLF Noise}

On several occasions it has been observed that short fractional-hop whistlers can trigger or enhance a burst of VLF noise. Examples of this at 46.3, 46.5 , and $49.4 \mathrm{sec}$ after 0605 UT on 6 December 1963 , are shown in figure 7 . There are two interesting features of the noise which is generated by such an interaction. When due allowance is made for the effects of the AGC following the intense short fractional-hop-whistle, the noise is seen to occur im- 

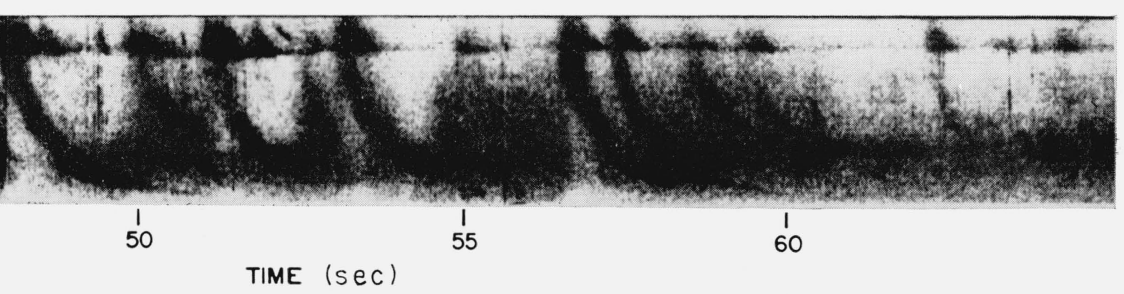

6 DECEMBER 1963 O605 U.T.

FIGURE 7. Spectrogram showing triggering of variable frequency noise band by short and long fractional hop whistlers. This recording was made at the Ottawa telemetry station. The satellite was on a magnetic shell $L=2.85$.

mediately after the whistler reaches the satellite. This indicates that the noise must originate at or below the satellite. The other striking feature of the triggered noise is that it has a well defined low frequency limit to its spectral distribution. It has been found that the cutoff frequency for this triggered noise often varies systematically with latitude in the same manner as the noise bands which have already been described. On occasion these noise bands are observed to grow uniformly out of the bursts of noise which are triggered by fractional-hop whistlers, also shown in figure 7 . At the low latitude limit for which these noise bands are observed, only short duration bursts of noise are triggered, but the duration of the triggered emissions increases with increasing latitude of the satellite until a continuous noise band is observed.

Not only is noise triggered by short fractional-hopwhistlers, but also by whistlers. Of particular interest is the fact that the lower cutoff frequency of the noise triggered by whistlers is the same as that triggered by short fractional-hop whistlers. Thus, electromagnetic waves traveling up a line of force, stimulate the same noise frequencies as waves traveling down the field line. These facts lead to two important conclusions. The noise bands observed by the satellite must originate in the immediate vicinity of the vehicle. Thus, the spectral characteristics of the noise must be determined in part by the medium in the vicinity of the satellite. In addition, there seems to be no strong reason why electromagnetic waves should trigger noise only at the height of the satellite. However, the satellite does not appear to observe triggered noise generated at other levels. This suggests that the VLF radiation which is stimulated on the field line on which the satellite is situated, does not propagate appreciable distances along this field line.

The short fractional hop whistler which occurred at $55.65 \mathrm{sec}$ after $0605 \mathrm{U}$ T is also interesting in that it shows a phenomenon associated with the ion gyrofrequency for protons in the plasma surrounding the satellite. The signal appears after the reception of the atmospheric at frequencies much less than 1 $\mathrm{kc} / \mathrm{s}$, shows initially a rapid rise in frequency, followed by a frequency which is nearly constant, and has previously been described [Smith et al., 1964] as being related to the ion gyrofrequency. While the relationship of this phenomenon suggests a "triggering" process, it appears possible that the noises are simply dispersed forms of the original atmospheric impulse (with dispersion arising from propagation from the ground to the satellite).

\section{Analysis of the Data}

In an effort to obtain more quantitative information on the noise bands which show a systematic frequency variation with the position of the space craft in the magnetic field, several of their features were scaled and plotted as functions of different parameters of the geomagnetic field. It was found that to a good approximation the frequency of the lower edge of the noise band was inversely proportional to the cube of the $L$ parameter at which the noise band was observed for $L$ values between 2.5 and 5. Since the cube of $L$ is inversely proportional to the magnetic field where the $L$ shell crosses the magnetic equator, the frequency of the lower edge of the noise band $f_{\text {low }}$ is proportional to the magnetic field $B_{0}$ or the gyrofrequency $f_{H}$ at the peak of the line of force on which the noise is observed. This fact is illustrated in figure 8 in which results from

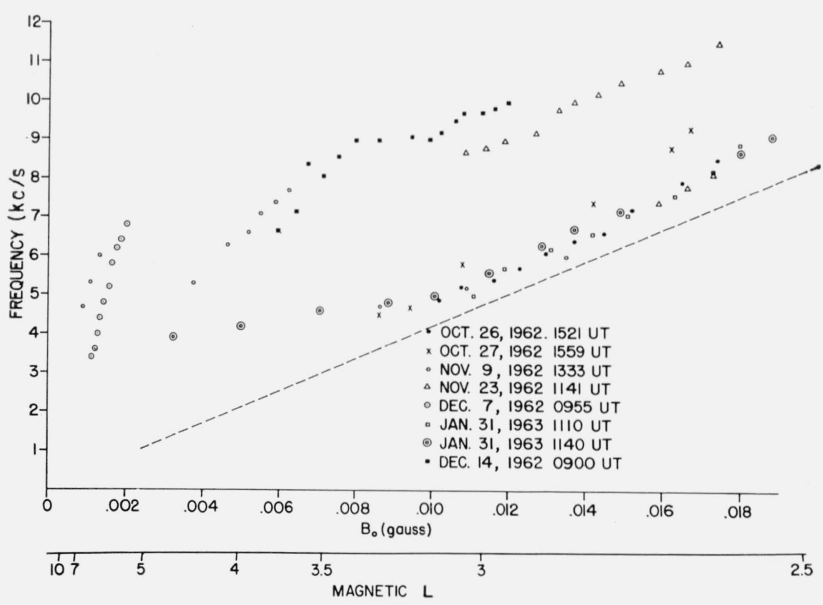

Figure 8. Plots of $\mathrm{f}_{\text {low }}$ (the lower edge of the noise band that shows systematic changes in spectral distribution with satellite latitude) versus $\mathrm{B}_{0}$ (the minimum value of the strength of the earth's field on the field line at which the noise was observed) for several different events.

The dashed line is drawn for $f=0.15 \quad\left(f_{H}\right)_{\min }$ (or $f=4.2 \times 10^{5} B_{0}$ ) which is discussed in the text. 
noise bands observed on several different occasions are combined. At high latitudes, or $B_{0}$ between 0.003 and 0.01 ( $L$ values of 3.1 to 4.7 ), $f_{\text {low }}$ varies approximately linearly with $B_{0}$ but shows no consistent trend from day to day. At lower latitudes $B_{0}$ between 0.01 and 0.02 ( $L$ values between 3.1 to 2.5) $f_{\text {low }}$ is not only proportional to $B_{0}$ but the slope of the points on the $\left(f_{\text {low }}, B_{0}\right)$ plots is about the same for all events. Moreover, in this latitude range the points appear to cluster about two frequencies separated by about $3.5 \mathrm{kc} / \mathrm{s}$.

At very high latitudes, or $B_{0}$ less than $0.002(L$ values between 5.5 to 7 ), a noise band is sometimes found which shows a systematic increase in frequency with decrease in latitude, but the slope of the points on the $\left(f_{\text {low }}, B_{0}\right)$ plot is very much steeper than at lower latitudes.

To a very high degree the noise observed at the same $L$ value in the northern and southern hemisphere is sometimes the same in spite of the fact that the observations in the two hemispheres are separated by at least $30 \mathrm{~min}$ in time and several degrees in magnetic longitude and the local gyrofrequencies at the points of the observations differ by as much as 30 percent.

The data of figure 8, except for the 31 January 1963 event, were all recorded at Prince Albert. The ground station recordings at Ottawa show that in all cases these noise bands were associated with major noise storms. The times of occurrence of these events, although not sufficient in number to be statistically significant, cluster about $10 \mathrm{U} T$, or $06 \mathrm{LMT}$, which is the time for the maximum of occurrence of $4 \mathrm{kc} / \mathrm{s}$ noise bursts observed by ground receivers at Ottawa, in our longer statistical study.

\section{Discussion}

All major noise storms are associated with Alouette hiss bands, although the hiss bands do not always show the characteristic systematic frequency variation. Recent studies indicate that emissions triggered by atmospherics are observed much more frequently than are hiss bursts or noise storms observed by ground receivers [Brice et al., 1964]. Out of 200 recordings presently being analyzed 90 show hiss bands. This section will discuss the possible relation of these hiss bands to current theories of VLF emissions.

Most theories of such emissions have centered around two resonances which are known to exist in the interaction of energetic particles with a stationary magnetoactive plasma [Brice, 1964]. In these resonances an EM wave is generated or amplified in the ambient plasma at a frequency which, due to the doppler affect, appears to the particles of the stream as zero frequency or the particle gyrofrequency. These resonances have been investigated by many workers both for the case of individual particles interacting with a plasma, for streams of particles, and for bunches of particles. Helliwell [1964] has concluded from these investigations that some degree of coherence or bunching of the particles is necessary to explain the amplitude of the VLF emissions ob- served on the ground. As yet it is not clear whether coherence is necessary to explain the noise bands observed by Alouette.

Dowden has examined the spectrum of the VLF radiation one might expect from the so-called traveling-wave-tube ('T'T) process. In this mechanism, a stream of electrons trapped in the earth's magnetic field forms an electron beam and the whistler mode which has a low velocity, acts as the anlog of the slow-wave structure of a traveling-wave tube. The frequencies which are amplified or generated by the 'TW' interaction must appear to the electrons of the beam as zero frequency. The condition for this to be true is the same as that required for Cerenkov radiation from a single particle viz

$$
V_{B}=\frac{C}{n \cos \theta}
$$

Where $V_{B}=$ beam velocity

$\theta=$ angle between the axis of beam and the wave normal of the emitted radiation $n=$ the refractive index of the medium for a wave of the amplified frequency with a propagation vector in the $\theta$ direction $C=$ velocity of light.

Figure 9 shows the results of a calculation based on this theory and a particular model of the electron density distribution in the exosphere. In this figure the interaction parameter $S / \lambda$ (which is proportional to the amplification of the 'TW'T mechanism in nepers is plotted for stream particles of small pitch angle guided along the field line terminating at a geomagnetic latitude of $60^{\circ}$. At other latitudes frequencies will scale so the maximum $S / \lambda$ for different latitudes occurs at the frequencies indicated by the dotted line of the figure.

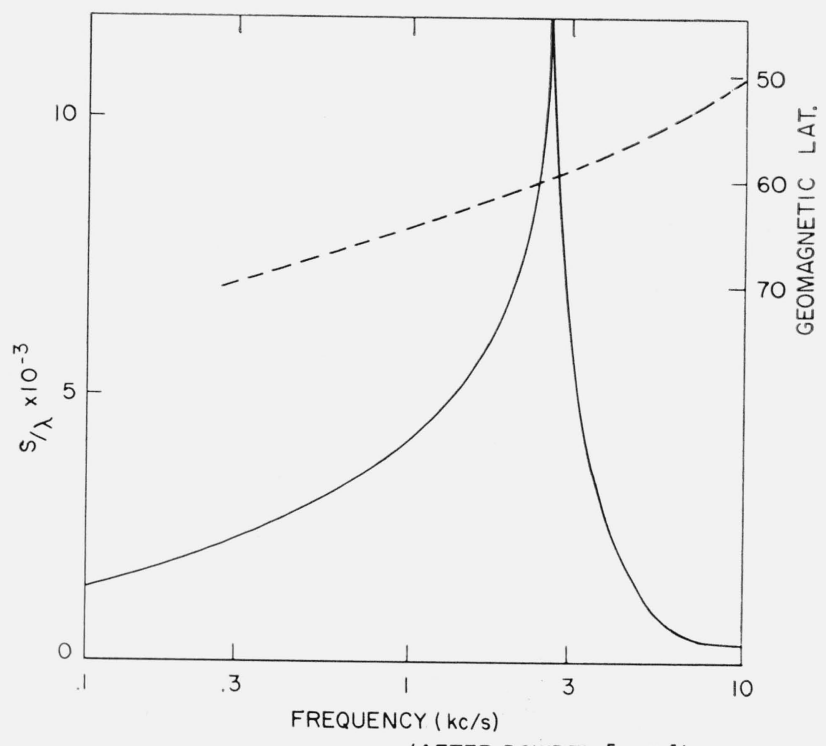

(AFTER DOWDEN [1962])

FIguRE 9. Amplitude-frequency spectra in nepers (which is proportional to the interaction parameter $\mathrm{s} / \lambda$ for the $T W T$ process as shown) for stream particles of small pitch guided along the field line terminating at geomagnetic latitude $60^{\circ}$. At other latitudes frequencies scale so that maximum amplification occurs at the frequency given by the dashed curve. 
This latitude dependence of the frequencies generated by the 'TW'T process has some of the features necessary to an explanation of the observed noise bands. For geomagnetic latitudes between about $65^{\circ}$ and $50^{\circ}$, the theory predicts that the frequencies most likely to be generated should range from about 1 to $10 \mathrm{kc} / \mathrm{s}$. The observed noise bands show a systematic frequency variation between 3 and 9 $\mathrm{kc} / \mathrm{s}$ for $L$ values between 3.5 and $2.5\left(\Lambda=56^{\circ}\right.$ to $51^{\circ}$ ). In addition the theory predicts that the variation with latitude of the frequency which experiences the greatest amplification is given approximately by the formula:

$$
f=0.15\left(f_{H}\right)_{\mathrm{min}}
$$

where $\left(f_{H}\right)_{\min }$ is the electron gyrofrequency at the top of the line of force on which the frequency is amplified. Such a formula closely approximates the frequency variation with latitude of a narrow band noise event such as that illustrated in figure 5 .

It is clear, however, from figure 9 that the frequency response of the 'TW'T process is not symmetrical, in that the amplification falls off much more slowly for low frequencies than for high frequencies. Thus, if a broad band of frequencies is generated on a given field line by a high flux of energetic particles, frequencies much lower than those given by the formula $f=0.15\left(f_{H}\right)_{\mathrm{min}}$ will be observed, whereas the noise band will not extend to frequencies much greater than those given by this expression. The observed noise bands do not support this prediction of the TWT mechanism. It is the low frequency edge of a broad band noise event such as that illustrated in figure 2 , which varies systematically with latitude and approximates to the formula $f=0.15\left(f_{H}\right)_{\mathrm{min}}$. Thus, the noise bands exhibit a cutoff effect which varies systematically with latitude, whereas the TWT process predicts no such cutoff effect.

The spectrum of the cyclotron radiation from highspeed electrons trapped on the earth's magnetic field lines does exhibit a low frequency cutoff [Dowden, 1963]. The radiation from a receding electron in the exosphere can be doppler shifted into the frequency band of the Alouette receiver, so that a stream of such electrons would produce band limited noise. Computed amplitude-frequency spectra for noise generated on field lines of $60^{\circ}$ and $63.5^{\circ}$ geomagnetic latitude by monoenergetic electron streams of $1 \mathrm{keV}, 6 \mathrm{keV}$, and $30 \mathrm{keV}$ are shown in figure 10 . These spectra exhibit the required feature that the low frequency cutoff does not change much with electron energy (except for high values of electron energy), whereas the upper frequency limit is quite variable. Unfortunately, the cutoff frequency is given approximately by $0.55\left(f_{H}\right)_{\min }$ and this differs by a factor of 3 from the cutoff frequencies which are observed, see figure 7 .

Both the TW'T process and the Doppler shifted cyclotron mechanism fail to explain the local nature of the noise bands observed by Alouette. As has already been noted the fact that whistlers and fractional-hop whistlers both trigger noise which has

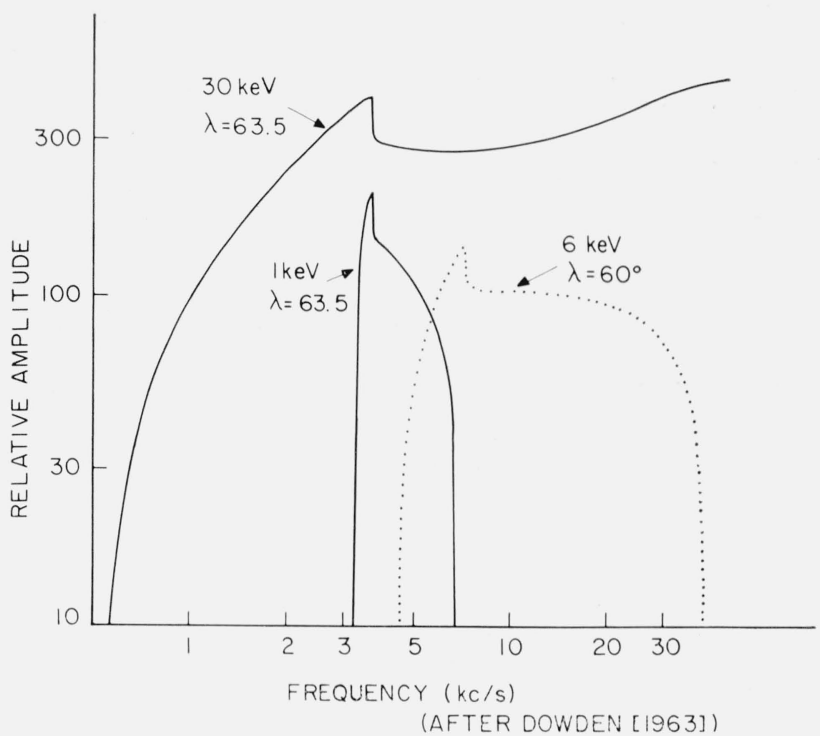

Figure 10. Amplitude-frequency spectra of hiss generated on field lines of $60^{\circ}$ and $63.5^{\circ}$ geomagnetic by the doppler shifted cyclotron processes by mono-energetic electron streams having a pitch angle distribution produced by isotropic injection in the equatorial plane.

A finite stream width would smooth out the discontinuity which occurs at 0.55 $\left(f_{H}\right)_{\min }$.

the same cutoff frequency as the Alouette noise bands suggest that the frequency cutoff is determined at least in part by the medium in the vicinity of the satellite. Moreover, the fact that there is no detectable time lag between the observation of the whistler and the noise which it triggers is strong evidence for the conclusion that this noise originates in the vicinity of the satellite. In the case of the Doppler shifted cyclotron mechanism the lowest observed frequency component originates on a given field line at the magnetic equator [Dowden, 1963]. In the case of the TWT mechanism; it is not as easy to specify the region of generation of the lowest observed frequency component found on a given field line. However, it is only under rather unusual circumstances that it would originate in the upper $F$ region. It has been suggested that this noise is related, not to ordinary VLF emissions as seen on the ground, but to an ion plasma oscillation, at the "lower hybrid" resonance, occurring close to the satellite [Brice and Smith, 1964]. This suggestion is currently being examined, but the results of this study will be the subject of another paper.

We thank W. E. Mather for operating the VLF ground station recorders, and for making the spectrograms from the satellite data, which are shown in this paper. We are also indebted to W. E. Thompson for the recordings made with the VLF noise burst receiver.

\section{References}

Barrington, R. E., and J. S. Belrose (1963), Preliminary reresults from the very-low frequency receiver aboard Canada's Alouette satellite, Nature 198, 651-656. 
Barrington, R. E., J. S. Belrose, and D. A. Keeley (Dec. 1963), VLF noise bands observed by the Alouette I satellite, J. Geophys. Res. 68, 6339.

Brice, N. M. (1964), Fundamentals of VLF emission generation mechanisms, J. Geophys. Res. 69, 4515-4522.

Brice, N. M., and R. L. Smith (1964), Observation of a VLF plasma resonance by Alouette I, Nature $\mathbf{2 0 3 ,} 926$.

N. M. Brice, R. L. Smith, J. S. Belrose, and Barrington, R. E. (1964), Triggered very low frequency emissions observed by the Alouette I satellite, Nature 203, 926-927

Dowden, R. L. (June 1962), Theory of generation of exospheric very-low-frequency noise (hiss), J. Geophys. Res. 67, $2223-2230$.

Dowden, R. L. (April 1963), Doppler shifted cyclotron generation of exospheric very-low-frequency noise (hiss), Planet. Space Sci. 11, 361-369.

Duncan, R. A., and G. R. A. Ellis (1959), Simultaneous occurrence of subvisual aurorae and radio noise on $4.6 \mathrm{kc} / \mathrm{s}$, Nature 183, 1618.

Ellis, G. R. A. (1959), Low frequency electromagnetic radiation associated with magnetic disturbances, Planet. Space Sci. 1, 253-259.

Finch, H. F., and B. R. Leaton (1957), The earth's main magnetic field-epoch 1955. Monthly Notices Roy. Astron. Soc. Geophysl Supl. 7 (6), 314-317.
Helliwell, R. A. (1964), Generation of VLF noise in the ionosphere by energetic particle streams, Space Res. 5, Proc. 5th Inter. Space Sci. Symp. Florence, 8-20 May 1964, (in press) (North Holland Pub. Co., Amsterdam).

Martin, L. H., R. A. Helliwell, and K. R. Marks (April 29, 1960), Association between Aurorae and VLF hiss observed at Byrd Station, Antarctica, SEL. Tech. Rept. No. 1, Stanford University, Stanford, Calif.

MeDiarmid, I. B., J. R. Burrows, E. E. Budzinski, and M. D. Wilson (Dec. 1963), Some average properties of the outer radiation zone at $1000 \mathrm{~km}$, Can. J. Phys. 41, 2064-2079.

McIlwain, C. E. (1961), Coordinates for mapping the distribution of magnetically trapped particles, J. Geophys. Res. 66, 3681 .

R. L. Smith, N. M. Brice, J. Katsufrakis, D. A. Gurnett, S. D. Shawhan, J. S. Belrose, and Barrington, R. E. (1964), An ion gyrofrequency phenomena observed in satellites Nature 204, 274.

Watts, J. M., J. A. Koch, and R. M. Gallet (1963), Observations and results from the "Hiss Recorder," and instrument to continuously observe VLF emissions, J. Res. NBS 67D (Radio Prop.), No. 5, 569-579.

(Paper 69D1-443) 